Rer. Sarde pübl., S. Paulo

$12: 95-43,1978$

\title{
RECURSOS PARA O COMBATE À TUBERCULOSE EM LONDRINA, PR, BRASIL
}

Lilia Bueno de Magalhães *

Nilton Tornero*

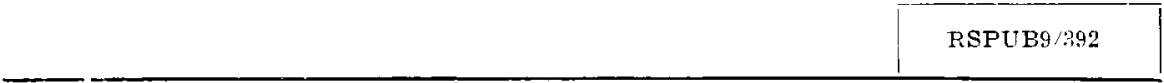

Magal hães, L. B. de \& TORNERO. N. Recursos para o combate à tuberculose em Londrina, PR, Brasil. Rev. Saúde públ., S. Paulo, 12:35-43, 1978.

Resumo: Por meio de informafoes obtidas em 1976. em visitas ao Dispensário de Tuberculose e ao Sanatório Noel Nutels, órgãos de combate à tuberculose no municipio de Londrina, PR, Brasil, foi discutida a atuaçào desses órgâos dentro de um sistema escalonado de prestaça de servicos. Foram comentadas várias atividades desses órgãos em relação às recomendaçóes da Divisão Nacional de Tuberculose. A incidência da tuberculose no municipio de Londrina foi calculada para anos recentes; foi caracterizada a procedencia dos casos novos diagnosticados em 1975-76. Critica-se particularmente o uso em massa da abrellgrafia. dando-se ênfase à baciloscopia para o diagnóstico e controle dos doentes.

Uniternos: Tuberculose, prevençao. Tuberculose, Londrina, PR. Brasil. Saúde pública, administração.

\section{INTRODUCAOO}

Segundo Cockburn \& Assaadi (1973) a tuberculuse é um dos maiores problemas de saude pública tanto para o conjunto dos paises desenvolvidos como para o conjunto dos países em desenvolvimentu. Aparece sistematicamente entre os dez maiores problemas de saúde pública em relação à morbidade nesses países; em relação à mortalidade varia entre a primeira e terceira causa de todos os óbitos conforme a região considerada para os países em desenvolvimento. e é a segunda causa entre as doenças transmissiveis nos paises desenvolvidos. Embora seja considerado problema de saúde pública no mundo todo, a magnitude varia conforme se considere os países em desenvolvimento e os desenvolvidus: enquanto que para um conjunto selecionado de paises desenvolvidos a taxa de mortalidade no biênio 1967-68 fui de 6,8/100.000 hab. para um conjunto de paises em desenvolvimento, foi de $34,3 / 100.000$ hah. Isto sem considerar a fidedignidade dos dados, principalmente no último conjunto de paises, onde, de maneira geral, os dados são subestimados.

Dados recentes da OMS (1976) mostram yue enquanto na América do Norte e Eurispa a incidência anual pode atingir 100 casos por 100 mil hab., esta mesma incidência é estimada em 200 para a América

* Do Departamento de Medicma Geral e Saud e Comuntária do Centro de Cienclas da Saude da Universidade Estadual de Londrina. Campus Unirersitário. Caixa Postal 2111 - Londrina, PR, Brasil. 
MAGALHAES, L. B. de \& TORNERO, N. Recursos para o combate à tubercu'sse em Londrina, PR, Brasil. Rev. Saúde públ., S. Paulo, 12:35-43, 1978.

Latina (América Central incluindo o México, América do Sul e Caribe), 250 para a Africa e 300 para algumas regiões da Ásia e Oceania. $\mathrm{Na}$ Europa chega a ser responsável por $2,8 \%$ do total de todos os óbitos em alguns países; esse valor sobe para 4,5\% nas Américas, atingindo máximos valores na Ásia (até 10,4\%). Quando se compara a América Latina com os Estados Unidos e Canadá, estima-se que a condição epidemiológica na América Latina é, no mínimo, dez vezes inferior; em algumas regiōes a situaçāo é pior agora do que antes da era da quimioterapia". O risco da infecção é calculado cem vezes superior no conjunto dos paises em desenvolvimento; isto se deve ao reservatório extremamente amplo nestes paises?.

Os dados disponiveis sobre o Brasil mostram que a situação é semelhante ao conjunto dos paises em desenvolvimento. Em 1975 foram notificados 30.260 casos $^{5}$, com uma incidência aproximada de 30 casos por 100 mil hab. Como outras doenças, o número de casos notificados variou com a região sem que, obrigatoriamente, os dados apresentados indiquem as áreas onde o problema é maior; o Nordeste notificou o maior número absoluto de casos (12.694), vindo a seguir a região Sudeste (9785 casos) dos quais somente São Paulo notificou 4.833 casos. Dados de 1969 , referentes às capitais, mostram que a mortalidade é de 38,5/100000 hab., representando $3,9 \%$ do total de todos os óbitos ${ }^{4}$. O reservatório bacilífero é considerado muito grande. Ao ingressarem na escola primária, $12 \%$ dos escolares são reativos ao teste tuberculínico. Em torno dos 20 anos, 50\% da população já se acha infectada pelo bacilo, o que contribui para a sua alta disseminação ${ }^{5}$. De 13 capitais estudadas, em somente 5 (Curitiba, São Paulo, Brasília, Cuiabá e Aracaju) os reatores fortes entre escolares à entrada da escola era menor que 10\%; em duas delas (Salvador e Belém) excedia $20 \% .^{3}$

A tuberculose frequientemente é lembrada como sendo uma doença de fácil diagnós- tico, tratamento eficaz e barato e medidas ce prevenção eficazes"-.i. Embora vários fatores influam no seu controle, este depende em grande escala dos recursos (materiais, humanos e financeiros) existentes. A fragilidade ou inexistência dos serviços sanitários na maioria dos países em desenvolvimento não permite que mesmo a tecnologia mais simples seja posta à disposição da maioria da população desses paises.

O presente trabalho visa estudar os recursos postos à disposição do combate à tuberculose em nível intermediário e discuti-los conforme as recomendações da Divisão Nacional de Tuberculose (DNT), dando-se ênfase aos aspectos administrativos.

\section{RECURSOS}

As informações aqui apresentadas foram obtidas em visitas, realizadas em fins de 1976, aos órgãos que participam do controle da Tuberculose no Municipio de Londrina - Dispensário de Tuberculose e Sanatório Noel Nutels - quando vários de seus funcionários foram entrevistados; outras informações também foram coletadas a partir dos registros existentes nesses órgãos.

1. Dispensário de Tuberculose. Englobado por Centro de Saúde da rede da Secretaria Estadual de Saúde (integração das atividades antituberculosas à rede de serviços gerais), o Dispensário funciona em prédio próprio desde 1967, atendendo os casos triados pelo INPS por meio de convênio. Desenvolve açōes preventivas, diagnósticas e curativas. Possui o seguinte pessoal (com as respectivas horas-trabalho/ semana): 3 médicos tisiologistas com $20 \mathrm{~h}$ cada um; 1 enfermeira com $30 \mathrm{~h} ; 2$ auxiliares de enfermagem com $30 \mathrm{~h}$ cada uma; 4 técnicos de Raios-X com $20 \mathrm{~h}$ cada um e 7 atendentes com $30 \mathrm{~h}$ cada um.

Em relação às ações preventivas é aplicada vacinação BCG oral em menores de 31 dias, tanto no Dispensário como nos Hospitais, pois o Dispensário fornece a vacina, a fim de que sua aplicação seja 
MAGALhães, L. B. de \& TORNERO. N. Recursos para o combate à tuberculose em Londrina, PR, Brasil. Rer, Saúde públ., S. Paulo, 12:35-43, 1978.

feita ainda no berçário. Para maiores de 31 dias a vacina usada é o BCG intradérmico, aplicada pelo Centro de Saúde, constituindo uma atividade integrada. Em 1975 foram aplicadas 5.508 vacinas com BCG intradérmico en crianças de 31 dias a 4 anos. Ainda en 1975, escolare: foram vacinados: de um total aproximado de 44.000 (incluindo as escolas publicas e particulares) vacinaram-se $37.626(86,0 \%)$, tendo se concentrado essas vacinaçoes no. meses de agosto a dezembro.

A maior demanda do Dispensário é o atendimento de pessoas que o procuram para tirar abreugrafias para carteiras de saúde, obedecendis exigèncias legais. A média nensal de abreugrafias está ao rector de 6.000 chapas. Em 1975 furan tiradas 74.639 abreugratias, sendo que 1.501 $(2,0 \%)$ sugeriam tuberculuse e destas, 244 $(0,3 \%$ do total) foram dadas comu pertencentes a pacientes com tuberculose.

O Dispensário deveria atender somente a região do 17" Distrito Sanitário com sede em Londrina e que engloba 20 municipios*. Pelo menos para 1975 até novembro de 1976, isto ocorreu com 91,7\% dos pacientes confirmados de tuberculose (casos novos) (Tabela 1). O 18" Distrito, com sede em Cornélio Procopio (60 $\mathrm{km}$ de Londrina) contribuiu com $4,3 \%$, vindo a seguir outros distritos en menor proporção. Dos $91,7 \%$ dos pacientes pertencentes ao 17\% Distrito, $70,0 \%$ provinham do próprio municipio de Londrina e $21,7 \%$ dos denais municípios que compojem o Distrito; em 4 desses municípios não se observou nenhum caso.

Para o diagnóstico usa-se 3 procedimentos: 19) abreugrafia; 2\%) baciloscopia do escarro feita no laboratório do Sanatório Noel Nutels); e $3^{\circ}$ ) PPD. Segundo os entrevistados, o Dispensário procura aplicar as recomendações da Divisão Nacional de Tuberculose en relação ao diagnóstico principalmente pela baciloscopia; porém, o primeiro procedimento diagnóstico usado é a abreugrafia porque existe o aparelho no Dispensário. O diagnóstico pela baciloscopia é feito somente em 3 Unidades Sanitárias do 17" Distrito: a de Rolândia, a de Bela Vista do Paraiso e a de Guaraci, que são Unidades integradas, existindo as ações anti-tuberculose como rotina e nāo como serviço especializado.

Estimou-se a incidência da tuberculose para o nunicipio de Londrina nos últimos anos (Tabela 2). Esta incidência variou de 58,9/100.000 hab. em 1976 até 77,8/100.000 hab. em 1974. Não se fez esses cálculos para o Distrito como um todo porque se deve admitir que pessoas residentes em cidades do $17^{\circ}$. Distrito possam procurar outros distritos para atendimento. Em relação a isto, é significativo o fato já citado de que em 1975-76 o Dispensário não diagnosticou casos novos provenientes de $4 \mathrm{mu}$ nicipios de sua jurisdiçâo. Não foi possivel o cálculo da prevalência; o número de casos existentes no último dia dos anos considerados, desprezando-se as procedências, foi o seguinte:

$$
\begin{aligned}
& \text { 1970: } 201 \text { casos } \\
& \text { 1971: } 210 \text { casos } \\
& \text { 1972: } 469 \text { casos } \\
& \text { 1973: } 456 \text { casos } \\
& \text { 1974: } 443 \text { casos } \\
& \text { 1975: } 364 \text { casos }
\end{aligned}
$$

En 1975 referiu-se 62 abandonos, o que dá uma taxa de $17 \%$. A inexistência de visitadoras agrava o problema; o Dispensário tem realizado uma média de 8 a 10 visitas ao mês, realizadas por outros profissionais.

Ainda em 1975 foram inscritos 634 comulnicantes, dos quais $597(94,2 \%)$ foram examinados; o controle c feito através do PPD nos menores de 15 anos e abreugrafia nos adultos.

O tratamento segue as recomendaçöes da Divisão Nacional de Tuberculose, com seus esquemas $\mathrm{A}$ e $\mathrm{B}$.

\footnotetext{
Portaria 149/70, da Secretaria de Saúde Pública. Departamento de Unidade Sanitária.
} 
MAGALHAES, L. B. de \& TORNERO. N. Recur sos para o combate à tuberculose em Londrina, PR, Brasil. Rev. Saúde públ., S. Paulo, 12:35-43, 1978.

TA B E L A 1

Procedéncia por Distrito Sanitário dos casos de tuberculose, inscritos no Dispensário Anti-Tuberculose de Londrina, PR, de janeiro de 1975 à novembro de 1976.

\begin{tabular}{|c|c|c|c|}
\hline Distrito & Sede & Freqüência & $\%$ \\
\hline $17^{\prime \prime}$ & $\begin{array}{l}\text { Londrina } \\
\text { Londring } \\
\text { Outros Muni- } \\
\text { cipios }\end{array}$ & $\begin{array}{l}373 \\
116\end{array}$ & $\begin{array}{l}70.0 \\
21.7\end{array}$ \\
\hline $18^{\prime \prime}$ & $\begin{array}{l}\text { Cornélio } \\
\text { Procópio }\end{array}$ & 23 & 4.3 \\
\hline $16^{\prime \prime}$ & Apucarana & 9 & 1.7 \\
\hline $15^{\prime \prime}$ & Maring'á & 6 & 1,1 \\
\hline $13 "$ & Cianorte & 2 & 0,4 \\
\hline $19^{\prime \prime}$ & Jacarezinho & 1 & 0,2 \\
\hline $11^{\prime \prime}$ & Campo Mourão & 1 & 0.2 \\
\hline 3 & Ponta Grossa & 1 & 0,2 \\
\hline- & Ignorado & 1 & 0,2 \\
\hline Total & - & 583 & 100.0 \\
\hline
\end{tabular}

TAEELA 2

Incidencia de tuberculose por 100 mil habitantes no municipio de Londrina, PR nos anos de 1970 a novembro de 1976.

\begin{tabular}{lccc}
\hline Ano & Populução $^{*}$ & Casos & $\begin{array}{c}\text { Coeficiente por } \\
\text { 100 mil hab. }\end{array}$ \\
\cline { 2 - 4 } 1970 & 230.328 & 154 & 66,9 \\
1971 & 239.878 & 165 & 68.8 \\
1972 & 249.428 & 181 & 72,6 \\
1973 & 258.978 & 168 & 64.8 \\
1974 & 268.528 & 209 & 77,8 \\
1975 & 278.078 & 190 & 68,3 \\
1976 & 286.832 & 169 & 58,9 \\
\hline
\end{tabular}

- Anos não censitários estimados pelo método aritmético, a partir dos dados dos censos de 1960 e 1970 .

O controle de tratamento é feitu pela baciloscopia mensal, até que a mesma se torne negativa, o que geralmente ocorre em torno do segundo mês. Se isto nāo ocorrer ainda 4 ou 5 meses após o início do tratamento, o caso é catalogado como resistente, passando-se a empregar o esquema B. Em relação ao controle, usa-se ainda a abreugrafia: após o diagnóstico é realizada de 4 em 4 meses e, por ocasião da alta, mais duas são feitas, sendo yue una delas fica nos arquivos do Dispensário e a outra é entregue ao paciente. $O$ controle de cura é feito por abreugrafia aos 6, 12 e 18 meses após a alta, sem obrigatoriedade.

$O s$ critérios de quimioprofilaxia seguenı as recomendaçōes da Divisão. A resistência às drogas - informada como pequena é diagnosticada clinicamente, não se empregando testes laboratoriais. 
MAGALHAES. L. B. de \& TORNERO. N. Recursos para o combate à tuberculose em Londina, PR. Brasil. Rev. Saude públ., S. Paulo, 12:35-4:3, 1978.

2. O Sanatirio No'l Nutels. Ao contrário do Dispensário, não pertence à Secretaria da Saúde, mas sim à Fundação Hospitalar do Paraná (FHP), entidade pública que possui vários hospitais próprios e mantém convênio com alguns outros. $O$ Sanatório é o unico existente no norte do Paraná; seu papel de órgão de referência e bem caracterizado: os dispensários existentes nessa região (norte do Paraná) enriam os casos a internar para o Sanatorio; eventualmente isto ocorre con pacientes de outros estados. Os critérios para internação são tanto clínicos (casos graves, emergências, resistência às drogas) quanto sociais (baixo nivel sócio-econômico).

O pessoal do Sanatório é o seguinte, com is respectivas horas-trabalho/semana: 7 médicos, com $20 \mathrm{~h}$ cada; 1 dentista com $24 \mathrm{~h}$; 1 assistente social com $24 \mathrm{~h} ; 1$ auxiliar de assistente social com $4 t h$; 1 auxiliar de enfermagem com $44 \mathrm{~h} ; 2$ operadores de Raio-X com 24 h cada; 32 atendentes con Ht h cada; 1 farmacêutico-bioquimico com 24 h; 3 técnicos de laboratório: 2 em 24 h e 1 em $44 \mathrm{~h} ; 1$ capelăo em $20 \mathrm{~h}$, e como diversus, 62 pessoas. Pussui ainda 7 irmãs cle caridade (1 técnico de enfermagem, 2 auxiliares de enfermagem, 3 atendentes de enfermagem e 1 atendente religiosa) que prestam serviços mediante convênio à parte; estão subordinadas à Diretoria de Enfermagem, que thes delegou a supervisão e algumas outras atividades da ala de Tisiologia.

O Sanatório Noel Nutels foi inaugurado em 1967 com 376 leitos. En 1.2.75 foi cclebrado um contrato de comodato com a Fundação Universidade Estadıal de Lundrina (FUEL) para a ocupação de parte do espaço fisioo dessa Fundação durante 5 anos. Dos 350 leitos disponiveis na época, u Sanatório ficou com 180 e o restante constituiu o Hospital Universitário Regional Norte do Paraná (HURNP). Várias atividades foran integradas com us recursos provenientes da FUEL. Como exemplo, " Sanatório não dispie de enfermagem própria (serviço) sendo o pessoal de enferma- gem subordinado ao serviço de enfermagem do HURNP; também o laboratório foi integrado, embora os laboratoristas do Sanatorio façam somente baciloscopias e sejam pagos pela FHP. Outros funcionários trabalham integrados tanto em atividades do Sanatório como do HURNP. Todo esse pessoal, porém, é pago pela FHP, cuia principal função é manter o Sanatório. A FHP libera una verba anual. cuja aplicaçào deve ser em material de consumo e serviços a terceiros.

Possui o Sanatório convênio com o INPS para internar doentes enviados por tal órgão. A diária paga inclui cama, mesa, médico e medicação de primeira linha. Outros procedimentos (exames laboratoriais e Raio-X) são pagos à parte à FHP e nāo ao Sanatório.

Dos 180 leitos, 102 são masculinos, dispostos em enfermarias de 8 leitos; os femininos e para crianças, en número de 78, estāo dispostos em enfermarias de $t$ leitos. Não há leitos reservados ao INPS: estão à disposição da demanda. $\mathrm{N}_{\text {() dia }}$ 17.11.76, por exemplo, 50 leitos foram ocupados por previdenciários e 105 por não contribuintes.

A Tabela 3 apresenta as taxas de ucupação e o tempo médio de permanència (em dias) para 1976. até no.embro. En relação à ocupação, os valores variaram de $7+\% \mathrm{em}$ agosto até $90 \%$ em outubro; o tenipo médio de permanência variou de 55,91 dias (abril) a 85,38 (setembro).

Em relação ao laboratorios - integradu - os técnicus yue realizan as baciluscupials atenden ao Sanatório, Dispensário e Huspital Universitário. Outros exames solicitados pelo pessoal do Sanatório são feitos no laboratório, pelos funcionários pagus pela FUEL. Faz-se uma média de 18 baciloscopias por dia pedidas pelo Dispensário e en torno de 20 pedidas pelo Sanatório. O HURNP pede uma média de 83 baciloscopias por mès, a maioria en material urinário, com poupuissima positiviciade, segundo declarou a informante. 
MAGALHĀES, L. B. de \& TORNERO, N. Recursos para o combate à tuberculose em Londrina, PR, Brasil. Rev. Saúde públ., S. Paulo, 12:35-43, 1978.

T A B E L A 3

Distribuição mensal da taxa de ocupação e do tempo médio de permanência dos doentes internados no Sanatório Noel Nutels de Londrina, PR, entre janeiro e novembro de 1976.

\begin{tabular}{lcc}
\hline Mês & $\begin{array}{c}\text { Taxa de } \\
\text { ocupação }(\%)\end{array}$ & $\begin{array}{c}\text { Tempo médio de } \\
\text { permanência em dias }\end{array}$ \\
\hline Janeiro & 85 & 75,58 \\
Fevereiro & 82 & 70,17 \\
Março & 81 & 75,51 \\
Abril & 84 & 55,91 \\
Maio & 88 & 80,95 \\
Junho & 89 & 68,25 \\
Julho & 80 & $\mathbf{7 7 , 5 7}$ \\
Agosto & 74 & 66,27 \\
Setembro & 82 & 85,38 \\
Outubro & 90 & 76,83 \\
Novembro & 87 & 81,42 \\
\end{tabular}
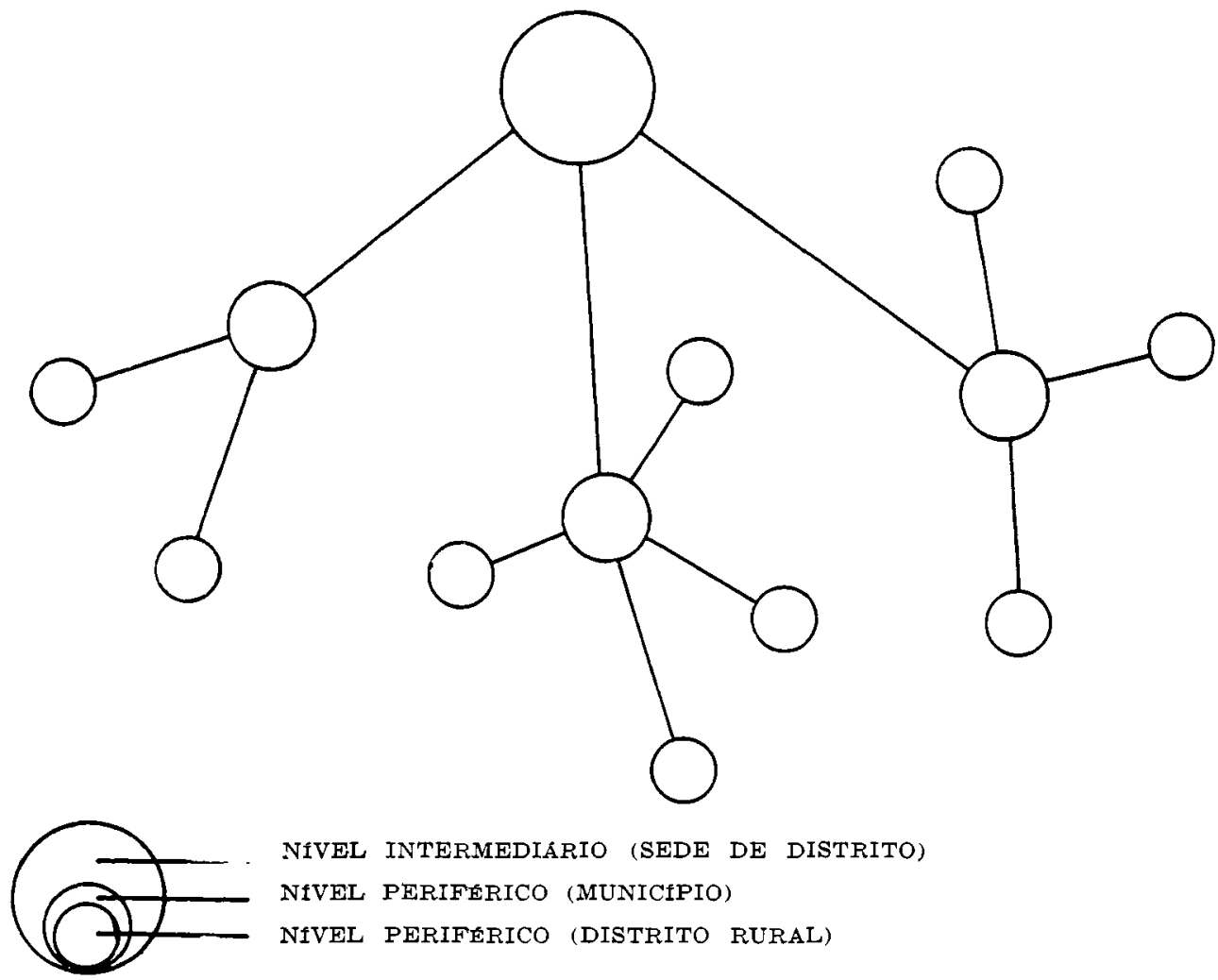

NAVEL INTERMEDIARIO (SEDE DE DISTRITO)

NIVEL PERIFERICO (MUNICIPIO)

NIVEL PERIFERICO (DISTRITO RURAL)

Figura

- Centralização grad

gradual da assistencia especializada

integração da assistência primária no combate à tuberculose. 
MAGALHAES, L. B. de \& TORNERO, N. Recursos para o combate à tuberculose em Londrina, PR, Brasil. Rev. Saúde puibl., S. Paulo, 12:35-43, 1978.

\section{COMENTARIOS}

A DNT, procurando racionalizar as atividades do setor, propõe que a ação anti-tuberculose seja exercida em 3 niveis; 0 periférico, o intermediário e o central ${ }^{1}$. 0 nível periférico (distrital e municipal) deve contar pelo menos com o agente local (auxiliar de saúde não especializado) e, se possivel, com um médico geral e um microscopista para realizar as baciloscopias. Estas Unidades polivalentes, integradas, periféricas, devem manter um fluxo constante com o nivel imediatamente superior, o nível intermediário, que deve, além de atender a demanda local, servir como referência a estas unidades periféricas (ver Figura). Para que isto ocorra, os recursos do nivel intermediário devem ser mais complexos; os serviços passam a ser especializados, com a existência de dispensários e hospitais. Recomenda-se tisiologistas, laboratoristas e radiologistas, entre outros. O nível central refere-se à coordenação geral.

Por constituir sede de distrito e com os recursos disponiveis, Londrina situa-se em nivel intermediário. Porém, sua ação é prejudicada por vários fatores: um deles, talvez o mais importante, é a precariedade da rede sanitária nos outros municipios: apesar de haver Unidades Sanitárias nos 20 municipios que compõem o Distrito, as atividades periféricas anti-tuberculose existem somente em 3 delas, excluindo-se Londrina. Como tal atividade não necessita obrigatoriamente de médico, conforme recomenda a DNT, deve-se tomar providências para que nas restantes Unidades existam pelo menos os agentes locais. A não-existência de uma infra-estrutura periférica acarreta o deslocamento dos pacientes em direção ao Dispensário, que deixa de operar como órgão de referência para atender toda a demanda do Distrito. A função para a qual está aparelhado - a de referência - fica extremamente prejudicada, ocorrendo uma situação típica de má utilização dos recursos existentes. Em relação a este último, um outro aspecto deve ser considerado: é o atendimento de pacientes provenientes de municipios pertencentes a outros distritos $(8,3 \%$ do total). Esta percentagem pode parecer pequena, porém, considerando-se uma procura de $8,0 \%$ provenientes de outros distritos, o Dispensário realizou, em 1975, um excesso de 6.000 abreugrafias, ou seja, um mês de trabalho foi gasto para atender pacientes oriundos de outros Distritos. A má utilização aumenta quando se sabe que todas as sedes de Distrito dos quais vêm pacientes para Londrina possuem aparelhos de abreugrafia. Atendendo área mal delimitada, o controle de muitos doentes torna-se impossivel. Logo, é oportuno estudar-se as causas porque os Dispensários dos demais distritos não estão atendendo suas demandas, permitindo a evasão. Esta evasão pode e deve estar, igualmente, ocorrendo para alguns municípios pertencentes ao Distrito de Londrina.

Para o Sanatório Noel Nutels os mesmos problemas citados acorrem, porém de maneira mais discreta, isto porque o Sanatório, sendo único no norte do Paraná, é Hospital de referência para toda essa região, englobando uma população estimada em 4,5 milhões de pessoas. O Hospital parece suficiente para atender essa demanda, tanto é que, dos 350 leitos, 170 foram cedidos à Universidade pelo contrato de comodato referido.

Falha grave detetada é a falta de visitadoras ou pessoal treinado para substitui-las - o que seria mais barato - e a conseqüente ausência de visitas. Sabe-se que uma das caracteristicas dos Dispensários é justamente a existência da atividade visita domiciliaria, diferenciando-o do ambulatório. Devido a esta atividade, o Dispensário está apto a procurar casos, ou seja, tem papel ativo no controle das doenças. Não existindo esta atividade, o Dispensário tem atuado como Ambulatório: atende unicamente a demanda passiva. Essa grave falha poderia ser corrigida em curto periodo, caso se treinasse atendentes para realizar tais visitas. 
MAGALHĀES. L. B. de \& TORNERO, N. Recursos para o combate à tiberculose em Londrina, PR. Brasil. Rer. Saúde publ., S. Paulo, 12:55-4\%, 1978.

Aspecto que não pode deixar de ser comentado é o uso excessivo de abreugrafias: pelas normas vigentes, um paciente que fica um ano em tratamento e faz o controle pós cura expõe-se a cerca de 9 abreugrafias em 2 anos e meio. O diagnóstico de nassa da tuberculose baseado em métodos radiográficos em geral, e abreugráticos em particular, constitui atualmente procedimento obsoleto. Tem sido condenado por vários autores e inclusive pela OMS. Senties e col.10 (1974) apontam vários inconvenientes no uso dos métodos radiofotográficos, principalmente para os paises em desenvolvimento, cujos recursos humanos, materiais e financeiros normalmente são reduzidos e as populaçōes dispersas: a) oferecen pouco acesso às populações suburbanas e rurais; b) procedimento ciclico, frequientemente único, não oferecendo continuidade na busca; c) requer o deslocamento dos suspeitos que em muitas ocasióes encontram-se a grandes distâncias; d) alto custo por descobrimento de doentes; e) baixo rendimento $(0,2 \%$ na população de $15 \mathrm{e}$ mais anos de idade), no caso específico do Dispensário de Londrina foi de $0,3 \%$; f) a maioria dos casos comprovados são sintomáticos; g) a manutenção das unidades é dificil e custosa; e h) a comprovação diagnóstica exige o exame da expectoraçāo. Entre nós, em recente e oportuno artigo, Gikovate e Nogueira: (1976) revisam " uso dos métrdos radiográficos em geral e da abreugrafia em narticular, no diagnóstico de massa da Tuberculose, com ènfase no Brasil, condenando tal procedimento. Afirmam que "a abreugrafia sistemática feita durante a infância, a puberdade e a idade da procriação indiscriminadamente, sem os cuidados acima citados (referem-se aos cuidados elementares com as radiaçies. raramente levados em conta), representa, sem duvida, uma dose excessiva de radiaçōes, desde que se tome em consideração o efeito cumulativo das mesmas". O método diagnóstico por excelência é a bacilos- copia, recomendada pela DNT. Senties e col."1" apontam as seguintes vantagens em relação à baciloscopia: a) acessibilidade a qualquer população urbana, suburbana ou rural (envolve a existência dos agentes locais); b) o procedimento é permanente; c) impede o deslocamento dos suspeitos, visto que se pode realizar no proprio local de residencia do interessado; (1) o custo de um microscópio é 60 vezes menor que 0 de uma unidade radiofotográfica; e) em qualquer lugar se pode coletar una amostra de expectoração nesmo por pessoal voluntáriu; e f) o procedimento estabelece 0 diagnóstico. Torna-se necessário revisar o uso de abreugrafias não só no seguinento de tuberculosos, como também como medida duagnóstica e cono rotina para carteiras de satide.

Finalmente, pelo que se pode sentir, a integração existe em vários setores, com evidentes beneficios na utilização dos recursos, sendo aceita como fator positivo pelo pessoal cum o qual se entrou em contato. Un dos fatores que facilita essa integração é que alguns tisiologistas pertencem tanto a) Dispensário (omo ar Sanatório, à pericia do INPS e ao corpo docente da Universidade.

\section{CONCLUSOES}

Do ponto de vista administrativo, as principais conclusies foram:

- O Dispensário tem sua açāo prejliclicacla como órgão de referência, a nivel intermediário. Isto se deve principalmente a dois fatores: precariedade da rede sanitária nos demais municipios do Distrito e atendimento da demanda proveniente de futers distritos.

- Pela ausência de visitação domiciliar, 1) Dispensário tem atendido a demanda passiva; tem operado mais como ambula- 
MAGAlHäs. L. B. de \& TORNERo. N. Recur sos para o combate à tuberculose em Londrina. PR. Brasil. Rev, Sande públ,, S. Paulo, 12:35-43, 1978.

torio afastando-se de seus propósitos, yue é a procura ativa dos casos.

- A integração sanitária, pelo menos até onde existe, foi referida como benéfica pelo pessoal entrevistado das Instituiçöes consideradas.

\section{AGRADECIMENTOS}

A todos os funcionários das instituiçōes visitadas pela boa vontade demonstrada no fornecimento das informações necessárias ao presente estudo.

MAgalhãEs, L. B. de \& TORNERO, N. IResoutres to face tuberculosis in Londrina, State of Paraná, Brazill Rev. Saúde públ., S. Pallo, 12:35-43, 1978.

ABSTRACT: At the end of 1976 the authors visited the Tuberculosis Dispensary and the Noel Nutels Sanatory, which are agencies against Tuberculosis in Lordrina, State of Parand, Brazil. Through the obtained information, they discuss the action of these agencies in an echeloned system of referral services; the former for the 1\%th Sanitary District, having its premises in Londrina, and the latter for the whole of the the North of Parana, in which it is the only existing Sanatory. Many routines of these agencies are discussed, compared with the recommendations of the National Division of Tuberculosis. The incidence of the disease in Londrina is calculated for the last few years; they characterize the origin of the new cases diagnosed in 1975-76; some other information is presented and commented. Criticism is particularly directed ct the use of mass roentgenphotography, giving emphasis to baciloscopy for the diagnosis and control of the disease.

UNITERMS: Tuberculosis prevention. Tuberculosis, Londrina, PR, Brazil. Public health administration.

\section{REFERENCIAS BIBLIOGRAFICAS}

1. AĈ̣A anti-tuberculose a nível periférico. Brasília. Ministério da Saúde/Ministério da Previdencia Social, 1974.

2. ACTIVIDADES de la OMS en 1975. Ginebra, OMS, 1976. p. 79. (Act. Org. mund. salud, 229).

ALmeida. A. P. de. Prevalência da infecção tuberculosa em escolares das capitais brasileiras. [Apresentado ao XVI Congresso Nacional de Tuberculose. João Pessoa, PB, 1972].

4. BOLETIM EPIDEMIOLógiCo. (Fundação SESP) Rio de Janciro, 6(4), 1974.

5. BOLETIM EPIDEMIOLóGico. (Fundação SESP) Rio de Janeiro, 8(10), 1976.

6. BRÓlIO, R. Quimioterapia da tuberculose. Rev. Saude publ,. S. Paulo. 9:71-85. 1975.
7. COCKBURN, W. C. \& ASSAAD, F. Some observations on the communicable diseases as public health problems. Bull. Wld Flth Org., 49:1-12. 1973.

8. Gikovate, $F$. \& NOGUEIRA. D. P. Abreugrafia sistemática em massa: inviabilidade economica e eventuais perigos de exposição às radiaçōes. Rev. Saúde públ., S. Paulo, 10:103-10, 1976.

9. PIO. A. \& WESTERN, K. Enfoque pars el control de la Tuberculosis en las Américas. Bol. Ofic. sarit, panamer, $80: 281-7,1976$.

10. SENTIES. V. R. et al. Bases del prosrama de control de la Tuberculosis en México. Bol. Ofic. sanit. panamer., 76 . $479-93,1974$.

Recebido para publicação em 16/05/19\% Aprovado para publicação em 14/0\%/19ri 